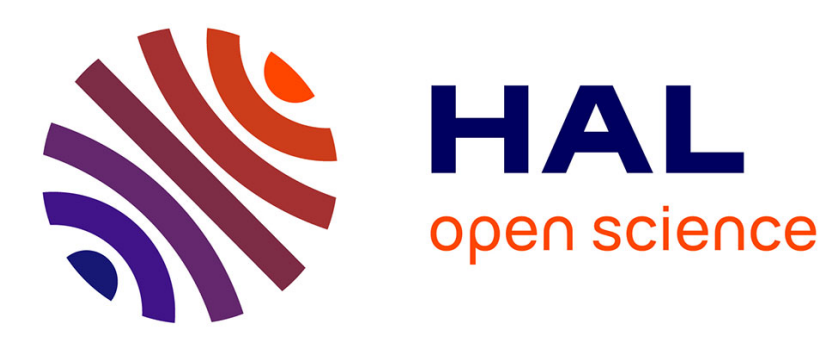

\title{
Constraints on the long-period moment-dip tradeoff for the Tohoku earthquake
}

\author{
V.C. Tsai, G.P. Hayes, Z. Duputel
}

\section{To cite this version:}

V.C. Tsai, G.P. Hayes, Z. Duputel. Constraints on the long-period moment-dip tradeoff for the Tohoku earthquake. Geophysical Research Letters, 2011, 38 (7), pp.L00G17. 10.1029/2011GL049129 . hal00878967

\section{HAL Id: hal-00878967 https://hal.science/hal-00878967}

Submitted on 16 Jun 2021

HAL is a multi-disciplinary open access archive for the deposit and dissemination of scientific research documents, whether they are published or not. The documents may come from teaching and research institutions in France or abroad, or from public or private research centers.
L'archive ouverte pluridisciplinaire HAL, est destinée au dépôt et à la diffusion de documents scientifiques de niveau recherche, publiés ou non, émanant des établissements d'enseignement et de recherche français ou étrangers, des laboratoires publics ou privés. 


\title{
Constraints on the long-period moment-dip tradeoff for the Tohoku earthquake
}

\author{
Victor C. Tsai, ${ }^{1,2}$ Gavin P. Hayes, ${ }^{2,3}$ and Zacharie Duputel ${ }^{4}$ \\ Received 1 August 2011; revised 19 September 2011; accepted 22 September 2011; published 25 October 2011.
}

[1] Since the work of Kanamori and Given (1981), it has been recognized that shallow, pure dip-slip earthquakes excite long-period surface waves such that it is difficult to independently constrain the moment $\left(M_{0}\right)$ and the dip $(\delta)$ of the source mechanism, with only the product $M_{0} \sin (2 \delta)$ being well constrained. Because of this, it is often assumed that the primary discrepancies between the moments of shallow, thrust earthquakes are due to this moment-dip tradeoff. In this work, we quantify how severe this momentdip tradeoff is depending on the depth of the earthquake, the station distribution, the closeness of the mechanism to pure dip-slip, and the quality of the data. We find that both longperiod Rayleigh and Love wave modes have moment-dip resolving power even for shallow events, especially when stations are close to certain azimuths with respect to mechanism strike and when source depth is well determined. We apply these results to USGS W phase inversions of the recent M9.0 Tohoku, Japan earthquake and estimate the likely uncertainties in dip and moment associated with the moment- dip tradeoff. After discussing some of the important sources of moment and dip error, we suggest two methods for potentially improving this uncertainty. Citation: Tsai, V. C., G. P. Hayes, and Z. Duputel (2011), Constraints on the long-period moment-dip tradeoff for the Tohoku earthquake, Geophys. Res. Lett., 38 , L00G17, doi:10.1029/2011GL049129.

\section{Introduction}

[2] Amongst the various earthquake source parameters, seismic moment $M_{0}$ (or moment magnitude $M_{w}$ ) is perhaps the parameter that is most utilized. However, for large earthquakes, short-period data become saturated and are therefore of limited use in determining total seismic moment. Long-period seismic waves therefore provide the best seismic constraints. Unfortunately, ever since the work of Kanamori and Given [1981], it has been recognized that, for shallow dip-slip earthquakes, these long-period data have poor sensitivity to $M_{0}$ as well as fault dip, $\delta$, with only the product $M_{0} \sin 2 \delta$ being well constrained. Despite this recognition, though, the moment-dip tradeoff has remained poorly quantified such that it is often unclear how large of an error it can represent. For example, the problem has been

\footnotetext{
${ }^{1}$ Seismological Laboratory, California Institute of Technology, Pasadena, California, USA.

${ }^{2}$ Geologic Hazards Science Center, U.S. Geological Survey, Golden, Colorado, USA.

${ }^{3}$ Synergetics Inc., Fort Collins, Colorado, USA.

${ }^{4}$ Institut de Physique du Globe de Strasbourg, Strasbourg, France.

Copyright 2011 by the American Geophysical Union. 0094-8276/11/2011GL049129
}

referred to numerous times but typically without quantification of how large related error is expected to be [e.g., Abercrombie et al., 2001; Kagan, 2003; Tsai et al., 2005; Kanamori, 2006; Konca et al., 2007; Hjorleifsdottir and Ekström, 2010; Hayes et al., 2011]. This motivates the current work; here we quantify the severity of the moment-dip tradeoff and how it depends on various key parameters. In the following sections, we first provide a summary of the normalmode theory necessary to understand the problem as well as a succinct restatement of the moment-dip tradeoff (section 2). Next, we examine the importance of source-station azimuth, station distribution in general, and source depth for momentdip errors (section 3). Finally, we suggest two methods that may help reduce these errors (section 4). Throughout this work, we use the USGS W phase inversion of the recent 11 March $2011 M_{w}=9.0$ Tohoku, Japan earthquake [Duputel et al., 2011; Hayes et al., 2011] as an important example of how our results can be applied.

\section{Normal Mode Theory and Restatement of the Moment-Dip Tradeoff}

[3] Within the context of standard normal mode theory, all displacement components can be expressed as

$$
u_{i}^{j}(r, \theta, \phi)=\sum_{l} y^{i j}(r)\left[\cos \left(\omega_{l} t\right) e^{-\omega_{l} t / 2 Q_{l}}-1\right] \mathbf{K}_{i}^{j}\left(\theta, \phi ; M_{0}, r_{s}, \phi_{s}, \delta, \lambda, l\right)
$$

where $u_{i}^{j}$ is the displacement of mode $j$ (either spheroidal, $j=\mathrm{S}$, or toroidal, $j=\mathrm{T})$ in the $i$ direction $(i=r, \theta$ or $\phi$ for spheroidal, $i=\theta$ or $\phi$ for toroidal), $\mathbf{K}_{i}^{j}$ are source excitation kernels (to be defined), and $y^{i j}$ are the normal mode radial eigenfunctions, e.g., as defined by Alterman et al. [1959], Ben-Menahem et al. [1970], Kanamori and Stewart [1976], and Kanamori and Given [1981]. (For example, $y^{r j} \equiv y_{1}$ as defined by Alterman et al. [1959] for the appropriate mode.) Here, $\mathbf{K}_{i}^{j}$ is assumed to be for a pure double couple source with scalar moment $M_{0}$, depth $d_{s} \equiv r_{\oplus}-r_{s}$, and fault parameters $\phi_{S}$ (strike), $\delta$ (fault dip), and $\lambda$ (rake). The coordinate system is assumed to be centered at the source, where $\theta$ is the angular distance from the source.

[4] Following Kanamori and Cipar [1974] and Kanamori and Stewart [1976], the excitation kernels can be written as

$$
\begin{aligned}
\frac{\mathbf{K}_{r}^{S}}{M_{0}}= & \hat{K}_{2} P_{l}^{2}\left[\sin \delta \cos \lambda \sin 2 \phi^{\prime}-\frac{1}{2} \sin 2 \delta \sin \lambda \cos 2 \phi^{\prime}\right] \\
& -\hat{K}_{1} P_{l}^{1}\left[\cos \delta \cos \lambda \cos \phi^{\prime}+\cos 2 \delta \sin \lambda \sin \phi^{\prime}\right] \\
& +\frac{1}{2} \hat{K}_{0} P_{l}^{0} \sin 2 \delta \sin \lambda
\end{aligned}
$$




$$
\begin{aligned}
\frac{\mathbf{K}_{\theta}^{S}}{M_{0}}= & \hat{K}_{2} \frac{d P_{l}^{2}}{d \theta}\left[\sin \delta \cos \lambda \sin 2 \phi^{\prime}-\frac{1}{2} \sin 2 \delta \sin \lambda \cos 2 \phi^{\prime}\right] \\
& -\hat{K}_{1} \frac{d P_{l}^{1}}{d \theta}\left[\cos \delta \cos \lambda \cos \phi^{\prime}+\cos 2 \delta \sin \lambda \sin \phi^{\prime}\right] \\
& +\frac{1}{2} \hat{K}_{0} \frac{d P_{l}^{0}}{d \theta} \sin 2 \delta \sin \lambda
\end{aligned}
$$

$$
\begin{aligned}
\frac{\mathbf{K}_{\phi}^{S}}{M_{0}}= & {\left[\hat{K}_{2} P_{l}^{2}\left(2 \sin \delta \cos \lambda \cos 2 \phi^{\prime}+\sin 2 \delta \sin \lambda \sin 2 \phi^{\prime}\right)\right.} \\
& \left.+\hat{K}_{1} P_{l}^{1}\left(\cos \delta \cos \lambda \sin \phi^{\prime}-\cos 2 \delta \sin \lambda \cos \phi^{\prime}\right)\right] / \sin \theta
\end{aligned}
$$

$$
\begin{aligned}
\frac{\mathbf{K}_{\theta}^{T}}{M_{0}}= & {\left[\hat{L}_{2} P_{l}^{2}\left(2 \sin \delta \cos \lambda \sin 2 \phi^{\prime}-\sin 2 \delta \sin \lambda \cos 2 \phi^{\prime}\right)\right.} \\
& \left.-\hat{L}_{1} P_{l}^{1}\left(\cos \delta \cos \lambda \cos \phi^{\prime}+\cos 2 \delta \sin \lambda \sin \phi^{\prime}\right)\right] / \sin \theta
\end{aligned}
$$

$$
\begin{aligned}
\frac{\mathbf{K}_{\phi}^{T}}{M_{0}}= & \hat{L}_{2} \frac{d P_{l}^{2}}{d \theta}\left[\sin \delta \cos \lambda \cos 2 \phi^{\prime}+\frac{1}{2} \sin 2 \delta \sin \lambda \sin 2 \phi^{\prime}\right] \\
& +\hat{L}_{1} \frac{d P_{l}^{1}}{d \theta}\left[\cos \delta \cos \lambda \sin \phi^{\prime}-\cos 2 \delta \sin \lambda \cos \phi^{\prime}\right]
\end{aligned}
$$

where $\phi^{\prime} \equiv \phi-\phi_{s}$, and $P_{l}^{m}(\theta)$ are associated Legendre functions. $K_{i}\left(r_{s}\right)$ are scaled versions of $y^{i S}$ and $\hat{L}_{i}\left(r_{s}\right)$ are scaled versions of $y^{i T}$, where $K_{i} \equiv y^{i S}(r) \hat{K}_{i}\left(r_{s}\right)$ and $L_{i} \equiv y^{i T}(r) \hat{L}_{i}\left(r_{s}\right)$ are as defined by Kanamori and Stewart [1976]. These definitions are such that $\hat{K}_{i}$ and $\hat{L}_{i}$ with odd subscripts are proportional to stress radial eigenfunctions whereas those with even subscripts contain dependencies on at least one displacement eigenfunction $\left(\hat{K}_{0}\right.$ also has a dependence on one of the stress eigenfunctions).

[5] For pure dip-slip events, $\lambda=\pi / 2$ so that, for example, equation (2a) simplifies to

$$
\frac{\mathbf{K}_{r}^{S}}{M_{0}}=-\frac{\hat{K}_{2}}{2} P_{l}^{2} \sin 2 \delta \cos 2 \phi^{\prime}+\frac{\hat{K}_{0}}{2} P_{l}^{0} \sin 2 \delta-\hat{K}_{1} P_{l}^{1} \cos 2 \delta \sin \phi^{\prime},
$$

and similarly, equations (2b)-(2e) would simplify to equations (3b)-(3e), respectively [not shown; henceforth, any mention of equation (3) refers to all five: (3a)-(3e)]. The basic moment-dip tradeoff for shallow dip-slip events can be understood by realizing that stress eigenfunctions must approach zero as $d_{s} \rightarrow 0$ (to satisfy stress free boundary conditions). This implies that $\lim _{d_{s} \rightarrow 0} \hat{K}_{\text {odd }} / \hat{K}_{\text {even }} \rightarrow 0$ and $\lim _{d_{s} \rightarrow 0} \hat{L}_{\text {odd }} / \hat{L}_{\text {even }} \rightarrow 0$ so that, in this case, all $\mathbf{K}_{i}^{j}$ in equation (3) are seen to be proportional to $M_{0} \sin 2 \delta$, with no other dependence on $\delta$ (e.g., no dependence on $\cos 2 \delta$ ). For this reason, it is sometimes stated that shallow dip-slip earthquakes can only resolve the product $M_{0} \sin 2 \delta$, and not $M_{0}$ and $\delta$ independently [Kanamori and Given, 1981].

\section{Sensitivity to the Moment-Dip Tradeoff}

[6] Given that all earthquakes occur at non-zero depth, $\hat{K}_{\text {odd }} / \hat{K}_{\text {even }} \neq 0$ and $\hat{L}_{\text {odd }} / \hat{L}_{\text {even }} \neq 0$, implying that even somewhat shallow events have non-negligible independent constraints on moment and dip. Especially with the improvement of modern seismic recordings, this fact suggests that it may be timely to reconsider the implications of the moment-dip tradeoff. In particular, in this work, we quantify the severity of the tradeoff to examine how it depends on a few key parameters, such as the average amplitude error and depth error. We apply these results to $\mathrm{W}$ phase inversions of the recent 11 March 2011 (M9.0) Tohoku earthquake to estimate likely errors.

\subsection{Raw Modal Sensitivity to Azimuth}

[7] For pure dip-slip $\left(\lambda=90^{\circ}\right)$ events, all sensitivities have similar dependencies on source-station azimuth $\left(\phi^{\prime} \equiv \phi-\phi_{s}\right)$, with the $A_{s} \equiv M_{0} \sin 2 \delta$ and $A_{c} \equiv M_{0} \cos 2 \delta$ terms of $\mathbf{K}_{i}^{j}$ either proportional to $\mathrm{C}_{0}+\mathrm{C}_{2} \cos 2 \phi^{\prime}$ and $\mathrm{C}_{1} \sin \phi^{\prime}$, respectively (for $\mathbf{K}_{r}^{S}, \mathbf{K}_{\theta}^{S}$, and $\mathbf{K}_{\theta}^{T}$ ), or proportional to $\mathrm{C}_{4} \sin 2 \phi^{\prime}$ and $\mathrm{C}_{3} \cos 2 \phi^{\prime}$, respectively (for $\mathbf{K}_{\phi}^{S}$ and $\mathbf{K}_{\phi}^{T}$ ), where $\mathrm{C}_{\mathrm{i}}$ are constants for a given mode, source depth and source-station distance. For example, for $\mathbf{K}_{r}^{S}$, equation (3a) shows that $C_{0}=\hat{K}_{0} P_{l}^{0} / 2, C_{1}=$ $-\hat{K}_{1} P_{l}^{1}$ and $C_{2}=-\hat{K}_{2} P_{l}^{2} / 2$. Since $\hat{K}_{\text {odd }} / \hat{K}_{\text {even }}$ and $\hat{L}_{\text {odd }} / \hat{L}_{\text {even }}$ are small for shallow sources, $C_{\text {odd }} / C_{\text {even }}$ can also be small, leading to decreased sensitivity to the $M_{0} \cos 2 \delta$ term.

[8] In many cases, one has abundant data to constrain all source parameters, and it is only important that one has a good enough station distribution and mode sampling (broad enough frequency bandwidth) to solve the inverse problem. The uncertainties inherent in this inverse problem will be discussed in later sections. However, it is sometimes useful to use a small set of modal data from a limited number of highquality stations to independently constrain $M_{0}$ and $\delta$ [e.g., Park et al., 2005]. In this case, even with only two independent observations at favorable azimuths, one can, in theory, uniquely determine $M_{0}$ and $\delta$ (if other source parameters are known), and the resolution is directly related to the source-station azimuths. If the $A_{c}$ term is the more difficult to resolve (given its smaller amplitude for a shallow source) and hence contributes most to the uncertainty, then stations located near the maxima of the $A_{c}$ terms while simultaneously being near the zeros of the $A_{S}$ terms will have the best relative resolving power for the moment-dip ambiguity. $\mathbf{K}_{r}^{S}$ and $\mathbf{K}_{\theta}^{S}$ will always have sensitivity to the $A_{s}$ term due to the non-zero $\mathrm{C}_{0}$ term (at least for relatively long-period waves), but $\mathbf{K}_{\phi}^{S}$, $\mathbf{K}_{\theta}^{T}$ and $\mathbf{K}_{\phi}^{T}$ have azimuths for which the $A_{s}$ coefficient is zero and the $A_{c}$ coefficient is non-zero. These special azimuths are $\phi^{\prime}=0^{\circ}$ and $180^{\circ}$ for $\mathbf{K}_{\phi}^{S}$ and $\mathbf{K}_{\phi}^{T}$ and $\phi^{\prime}= \pm 45^{\circ}$ and $135^{\circ}$ for $\mathbf{K}_{\theta}^{T}$, where $\phi^{\prime}$ is azimuth with respect to the strike of the rupture source. For these azimuths, normal-mode amplitudes are sensitive only to $M_{0} \cos 2 \delta$. Of course, in order to know which azimuths $\phi$ correspond to $\phi^{\prime}$, one must have prior knowledge of $\phi_{s}$ (or determine it jointly). One may also note that $\mathbf{K}_{\phi}^{S}$ (spheroidal mode contribution to transversely polarized waves) is expected to be smaller than $\mathbf{K}_{\theta}^{S}$ for all but the lowest-order (low $l$ ) modes due to the dependence on derivatives of $P_{l}^{m}$.

\subsection{Amplitude to Moment/Dip Error (for W Phase)}

[9] Each modal sensitivity $\mathbf{K}_{i}^{j}$ depends on source-station azimuth as described in section 3.1 and depends on sourcestation distance through its dependence on $P_{l}^{m}$. The total sensitivity of a particular phase can then be determined by summing the individual modal contributions to the total displacement of the phase. Here, we use W phase [Kanamori, 1993; Kanamori and Rivera, 2008; Duputel et al., 2011] observations of the recent 11 March 2011 Tohoku, Japan earthquake as an important example of long-period obser- 


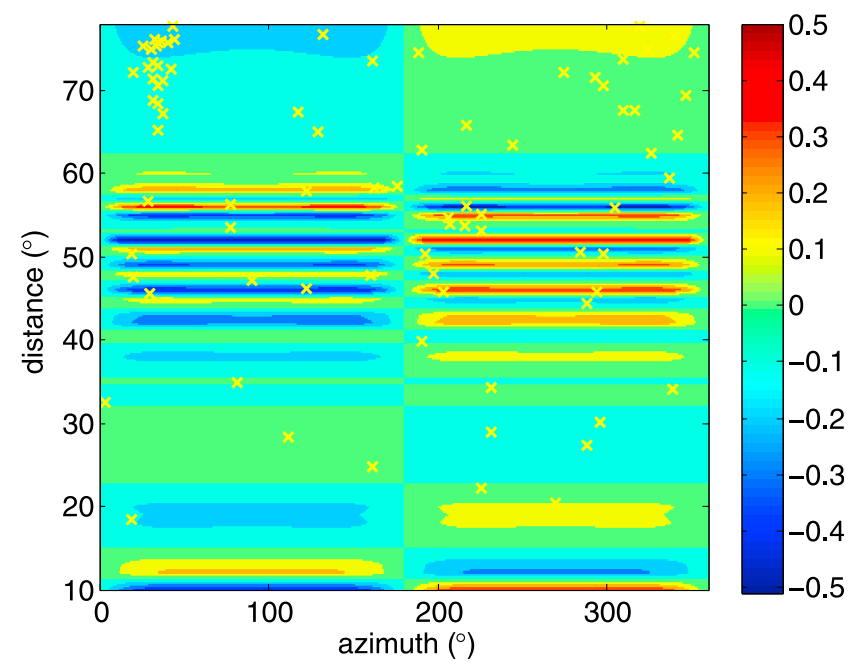

Figure 1. $\mathrm{A}_{\cos 2 \delta} / \mathrm{A}$ for $\mathrm{W}$ phase LHZ Amplitudes as a function of $\phi^{\prime}$ and $\theta$. The station distribution relative to the Tohoku, Japan earthquake location and strike are plotted as yellow crosses.

vations for which the concepts addressed can be applied. We note that $\mathrm{W}$ phase can be thought of as a superposition of higher-order Rayleigh waves with a group velocity slightly slower than the P wave, or alternatively as a superposition of long-period P, PP, SP, PS, etc. body waves.

[10] We perform a synthetic normal-mode summation for the USGS W phase mechanism $\left(d_{s} \approx 24 \mathrm{~km}, \delta \approx 14^{\circ}\right)$ and consider a (200s-1000 s) bandpassed W phase window (that immediately follows the $\mathrm{P}$ wave but arrives distinctly before the main S-wave arrival). From these data, we obtain W phase amplitudes that agree well with the azimuthal variability expected of equation (3) and discussed in section 3.1. Moreover, the variability with distance is also as expected for a superposition of a few higher-order Rayleigh waves. To provide an idea of the relative sensitivities to the $A_{c}$ term, in Figure 1 we plot the vertical-component (LHZ) best-fitting amplitudes of the sin $\phi^{\prime}$ term divided by the total LHZ amplitude. This amplitude ratio provides the relative sensitivity of vertical-component $\mathrm{W}$ phase amplitudes to the $A_{c}$ term compared with the total sensitivity. As shown, this ratio is typically of order $\approx \pm 0.2$ within the main $\mathrm{W}$ phase window (distances of $15^{\circ}$ to $90^{\circ}$ ), showing the generally weaker sensitivity to $A_{c}$ for shallow subduction earthquakes, but is occasionally quite large (up to \pm 0.5 , e.g., at a distance of $52^{\circ}$ ).

[11] To quantify the sensitivity of the USGS W phase solution to $M_{0}$ and $\delta$, we compare the W phase amplitude data with $\mathrm{W}$ phase synthetic data at each station. The data show that the average maximum $\mathrm{W}$ phase amplitudes is $2.6 \mathrm{~mm}$ and comparison to synthetics shows an average standard deviation of $0.50 \mathrm{~mm}$ (using only components selected in the original $\mathrm{W}$ phase solution). Taking these statistics as representative, and performing ten thousand independent realizations using amplitudes with these statistical properties, we estimate a standard deviation of $\varepsilon_{A s}=3.02 \%$ for the $A_{s} \equiv M_{0}$ $\sin 2 \delta$ amplitude and a standard deviation of $\varepsilon_{A C}=11.5 \%$ for the $A_{c} \equiv M_{0} \cos 2 \delta$ amplitude. Since $M_{0}$ and $\delta$ can be solved for in terms of these two amplitudes as $M_{0}=\sqrt{A_{s}^{2}+A_{c}^{2}}$ and
$2 \delta=\tan ^{-1}\left(A_{s} / A_{c}\right)$, we can then solve for deviations in $M_{0}$ and $\delta$, as

$$
\frac{\Delta M}{M_{0}} \approx \sin ^{2} 2 \delta_{0} \frac{\Delta A_{s}}{A_{s}}+\cos ^{2} 2 \delta_{0} \frac{\Delta A_{c}}{A_{c}} \text { and } \frac{\Delta \delta}{\delta_{0}} \approx \frac{\Delta A_{s}}{A_{s}}-\frac{\Delta A_{c}}{A_{c}}
$$

where it is assumed that $\delta_{0} \ll 1$ to simplify equation (4). Uncertainties can similarly be calculated and this results in a standard deviation for $M_{0}$ of $9.0 \%$ and a standard deviation for $\delta$ of $11.9 \%$. Pure amplitude errors therefore account for 0.037 magnitude units of (one-sigma) uncertainty and $1.7^{\circ}$ of dip uncertainty in the USGS W phase solution for the Tohoku earthquake.

\subsection{Depth to Moment/Dip Error}

[12] In addition to the effect of pure amplitude errors, errors in source depth will cause significant, non-random uncertainties in $M_{0}$ and $\delta$. Considering only shallow events $\left(d_{s} \leq\right.$ $40 \mathrm{~km})$, the sensitivity function ratios $\hat{K}_{\text {odd }} / \hat{K}_{\text {even }}$ and $\hat{L}_{\text {odd }} / \hat{L}_{\text {even }}$ can be approximated as linear with depth. (This neglects any sharp boundaries in elastic structure, which cause jumps in the sensitivity functions.) With this approximation, we can describe the error due to an incorrect depth by

$$
\Delta\left[d_{s} M_{0} \cos 2 \delta\right]=0 \text { and } \Delta\left[M_{0} \sin 2 \delta\right]=0
$$

where $\Delta[\cdot]$ denotes the variation in $[\cdot]$. Equation (5) can be solved algebraically for $\Delta \mathrm{M}$ and $\Delta \delta$ for a given $\Delta d_{s}$. When $\delta_{0} \ll 1$, this expression simplifies to

$$
\frac{\Delta M}{M_{0}} \approx-\frac{\Delta \delta}{\delta_{0}} \approx-\cos ^{2} 2 \delta_{0} \cdot \frac{\Delta d_{s}}{d_{s 0}}
$$

where the small-angle approximation $\cos \mathrm{x} \approx 1-\mathrm{x}^{2} / 2$ has been used. As one example, if the true depth were $30 \mathrm{~km}$ and the model depth were $25 \mathrm{~km}\left(\Delta d_{s}=-5 \mathrm{~km}\right)$, then equation (6) would imply that the moment is over-estimated by about $13 \%$ and the dip is under-estimated by the same amount (using $\delta_{0}$ of $14^{\circ}$ ). Similarly, if the model depth were $35 \mathrm{~km}$ compared with a true depth of $30 \mathrm{~km}$, moment would be underestimated by about $13 \%$ and the dip would be overestimated by the same amount. This moment-dip-depth bias can explain some features of synthetic tests we have performed (see Figure 2) in which we fix the $\mathrm{W}$ phase solution at different depths within the upper and lower crust of PREM [Dziewonski and Anderson, 1981]. In these tests, the firstorder differences in moment and dip within a given PREM layer are explained well by equation (5). However, crossing PREM interfaces (which are ignored in equation (5)) results in more complex variations for which the simple approximation discussed above does not hold (see Figure 2).

[13] When independent constraints on depth are not available, these moment-dip-depth errors depend on the depth sensitivities inherent in equation (2). Unfortunately, inversions based on long-period modes have poor sensitivity to depth for shallow earthquakes [e.g., Dahlen, 1993]. This poor sensitivity results from the fact that for such events, $K_{\text {even }}$ and $\hat{L}_{\text {even }}$ have relatively small variations with depth [e.g., Ben-Menahem et al., 1970] and therefore most of the sensitivity to source depth is through the product $d_{s} M_{0} \cos 2 \delta$ term described in equation (5). We note that the $\hat{K}_{\text {even }}$ and $\hat{L}_{\text {even }}$ 


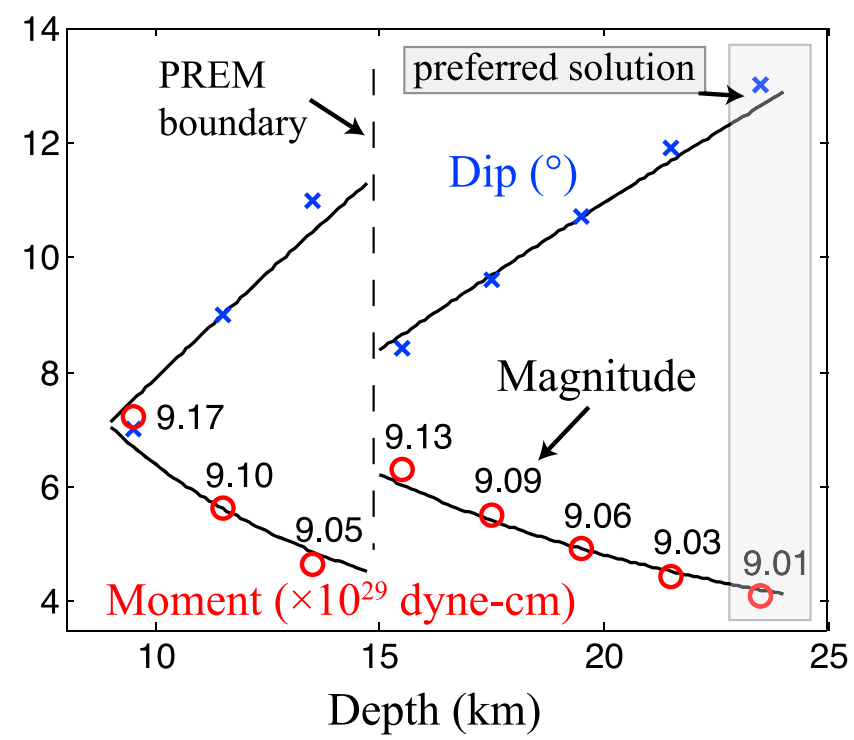

Figure 2. Moment-dip-depth bias for the Tohoku USGS W phase solution for depths within the upper crust $\left(d_{s} \leq 15 \mathrm{~km}\right)$ and lower crust $\left(15 \leq d_{s} \leq 24.5 \mathrm{~km}\right)$ of PREM. The eight different solutions considered use the same data but use depths fixed to different values between 9 and $24 \mathrm{~km}$. As shown, the resolved dips (blue crosses) and moments (red circles) are significantly affected by the choice of fixed depth, and most of this variability within a given layer of PREM is as predicted by equation (5) (black lines). We note that the misfits of all solutions shown are quite similar (with the best-fit solution at $23.5 \mathrm{~km}$, shaded in gray, having a misfit differing by less than $5 \%$ from that of the worst-fit solution at $9.5 \mathrm{~km})$.

terms do have non-negligible sensitivity to $d_{s}$, so that $d_{s}$ does not completely trade off with $M_{0} \cos 2 \delta$.

\section{Reducing the Moment-Dip Ambiguity}

[14] Given the importance of good constraints on seismic moment and source dip, it is of interest whether there may exist methods to reduce the errors discussed in previous sections. Here, we provide preliminary analyses of two ways in which improved data processing may help reduce the errors discussed in section 3.2 and section 3.3, respectively.

\subsection{Reducing Amplitude Errors}

[15] We first discuss whether it is possible to reduce the average amplitude errors $\left(\Delta A_{s}\right.$ and $\left.\Delta A_{c}\right)$ discussed in section 3.2. If each amplitude measurement is independent of all others and errors are uncorrelated with constant variance, it can be proven that standard regression provides an unbiased estimate of the model parameters [e.g., Fox, 1997], which, in this case, are the amplitudes $A_{s}$ and $A_{c}$. However, if there are significant modeling errors that result in spatially correlated errors, then improvements can theoretically be made. Such model errors are likely in abundance for typical long-period inversion schemes like the $\mathrm{W}$ phase method. For example, PREM [Dziewonski and Anderson, 1981] and other 1D velocity models are widely used as models for the normal mode summations [e.g., Kanamori and Rivera, 2008] despite the fact that 3D structure is known to significantly modify the waveforms. These deficiencies result in spatially correlated errors at a variety of wavelengths related to the length scales of the dominant structures affecting the waveforms, and are observed in $\mathrm{W}$ phase inversions. To account for these spatially correlated errors, it would be advantageous to down-weight not only data from regions with highly correlated data, but to also down-weight data from regions that are not expected to contribute significantly to resolving the desired parameters. To account for these two ideas, we suggest (1) weighting data to simulate evenly spaced station coverage, and (2) further weighting data to favor stations with larger expected $A_{c}$ and $A_{s}$ amplitudes.

[16] In this work, we provide preliminary synthetic analysis that suggests both of these weighting schemes can be useful. Again using a moment tensor solution that resembles the USGS W phase Tohoku earthquake solution, we build a synthetic dataset produced from standard normal-mode synthetics but with perturbations added to the $\mathrm{W}$ phase amplitudes in a spatially correlated fashion. We then invert this synthetic dataset using 3 different weighting schemes (i), (ii), and (iii). In (i), all stations are equally weighted (the traditional method); in (ii), stations are weighted inversely to the density of stations within a set of 40 Gaussians evenly spaced in azimuth and source-station distance; in (iii), stations are also weighted inversely to the density of stations but have an additional weighting factor proportional to total $\mathrm{W}$ phase amplitude. Scheme (ii) is therefore expected to account for (1), whereas scheme (iii) should account for both (1) and (2). Schemes (ii) and (iii) require an a priori estimate of location, and scheme (iii) also requires an estimate of the focal mechanism; in these tests, we use scheme (i) to provide this information and an iterative approach could potentially be used. W phase inversion results (represented as a best-fitting double couple) using (i), (ii) and (iii) are given in Table 1 and are compared with the 'true' synthetic values. As shown, (ii) produces an improvement in all fault source parameters compared with (i), and thus clearly demonstrates the potential usefulness of weighting towards a more uniform station coverage. Comparing (ii) and (iii), we observe that (iii) improves upon $M_{0}$ (and probably also $\delta$ at an undetectable level) but at the cost of larger errors in $\phi$ and $\lambda$. It is currently unclear whether this behavior is general or not, but it at least suggests that improved sensitivity to $M_{0}$ could be gained at the cost of decreased sensitivity to other parameters. Substantially more testing must be done to determine optimal weights.

\subsection{Reducing Depth Errors}

[17] The second question we discuss is whether depth errors can be reduced. In the absence of any independent

Table 1. Best-Fitting Double Couple Inversion Results for Synthetic Dataset ${ }^{\mathrm{a}}$

\begin{tabular}{lcccc}
\hline & $M_{0}$ & $\delta\left(^{\circ}\right)$ & $\phi\left(^{\circ}\right)$ & $\lambda\left({ }^{\circ}\right)$ \\
\hline Input ("truth") & 5.5076 & 9.4 & 193.6 & 81.1 \\
Scheme (i) & 5.2120 & 8.9 & 202.1 & 87.0 \\
Scheme (ii) & 5.2291 & 9.2 & 198.0 & 86.7 \\
Scheme (iii) & 5.2484 & 9.2 & 198.6 & 87.4 \\
\hline
\end{tabular}

${ }^{\text {a}}$ The first line ("Input") shows the true input synthetic values. Scheme (i) uses uniform station weighting; scheme (ii) uses weights inversely proportional to station density; scheme (iii) uses weights as in (ii) but which are also weakly weighted towards higher expected W-phase amplitudes. 

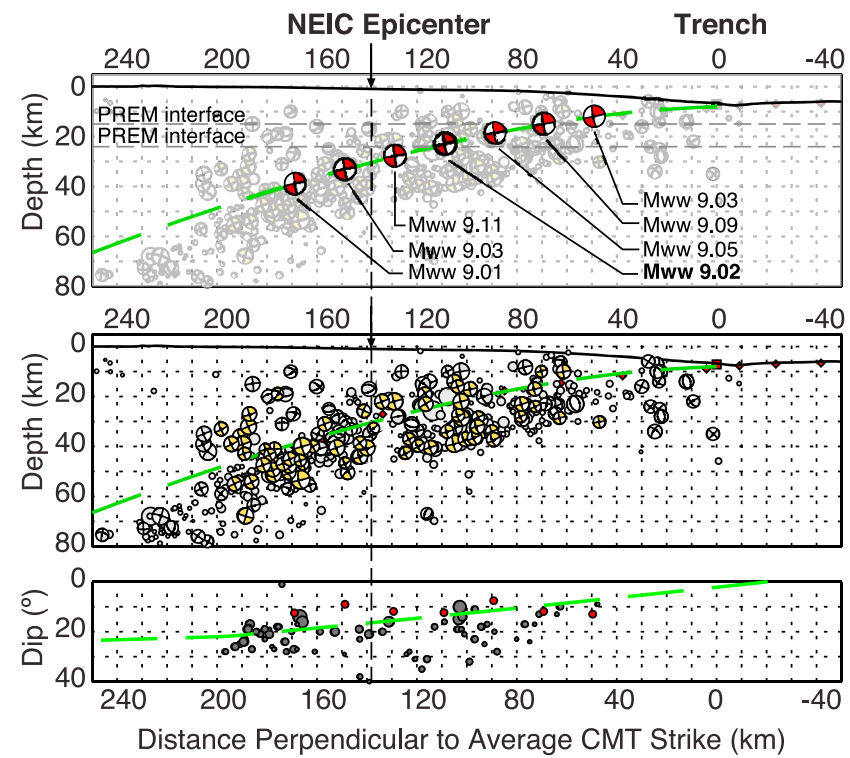

Figure 3. W phase inversions for the 11 March 2011 Tohoku earthquake informed by a priori knowledge of the 3D geometry of the Pacific plate beneath Japan, using the USGS Slab1.0 model [Hayes et al., 2009]. (top) A series of $\mathrm{W}$ phase inversions for the CMT solution of this earthquake at fixed locations along the slab interface. The preferred solution, in maroon, produces the best fit to 200 globallydistributed waveforms, and also best recovers the dip of the slab interface at that location (see Figure 3, bottom). (middle) The data used to constrain the slab interface in this region. Yellow CMTs represent gCMT solutions plotted at their EHB [Engdahl et al., 1998] hypocenters. Grey CMTs and circles are background seismicity, not used for constraint because they are too small to have associated gCMT solutions, or because their source mechanism is not subductionthrust-related. Red diamonds represent interpretations of the interface from local active seismic data [Miura et al., 2005], also used to aid geometry constraint. The red square marks the trench location interpreted from GEBCO 2008 bathymetry. The green dashed line represents the Slab1.0 interface. (bottom) The dips of the individual mechanisms used to constrain the geometry of the slab interface (gray dots; yellow CMTs in Figure 3, middle), overlain with the dips of the fixed-centroid CMT inversions from Figure 3 (top).

depth constraints, it would be useful to have better depth resolution than is possible by using the long-period data alone. Here, we suggest one possible approach to improving this resolution by utilizing a priori knowledge of the slab geometry for subduction zone earthquakes. Using the USGS Slab1.0 3D subduction geometry model [Hayes et al., 2009], or other regional models [e.g., Hashimoto et al., 2004], we can derive a robust estimate of nucleation depth for subduction interface events based on the preliminary epicenter of the earthquake. Similarly, the variation of slab geometry in the model over the source region surrounding the hypocenter informs us of the expected range in centroid depth, and thus allows us to constrain depth estimates to narrower bounds than would otherwise be possible.

[18] We test this methodology for the Tohoku earthquake by inverting for the W phase CMT solution at a selection of fixed locations along the slab surface, in a direction perpen- dicular to the strike of the slab at the PDE epicenter, and a spacing of $10 \mathrm{~km}$ horizontally (the approximate resolution of W phase Green's functions). At each location, we examine the goodness of fit of the inversion, as measured by RMS misfit criteria, as well as how well the inversion recovers the actual parameters of the slab at that location (see Figure 3). Our preferred solution, marked in maroon on Figure 3, has both the smallest RMS misfit and closest best-fitting double couple dip when compared to the inferred slab interface. Furthermore, this solution matches the depth of the published USGS W phase solution, is similar to the depth of the gCMT [Dziewonski et al., 1981; Ekström et al., 2005] solution $(20 \mathrm{~km})$, and also corresponds well to the preferred solution from Figure 2. The result matches what we know about the predominantly up-dip rupture of this earthquake [e.g., Hayes, 2011], with the added benefit that it inherently matches tectonic models of the region. Finally, we note that for an average slab-interface dip of $14^{\circ}$, a horizontal location error of $20 \mathrm{~km}$ would correspond to a depth error of only $5.0 \mathrm{~km}$. On the other hand, the unconstrained long-period solutions span a wide range of depths $(\approx 15-40 \mathrm{~km})$ for which the RMS misfit is relatively close to the minimum RMS misfit, and therefore which satisfactorily fit the data. Use of the a priori slab interface estimate therefore helps to reduce the range of likely depths, which then allows for a more robust constraint on moment and dip.

\section{Conclusions}

[19] We have discussed and quantified the moment-dip tradeoff inherent in earthquake CMT inversions, and have shown that it depends on a number of key parameters. For example, the severity of the tradeoff depends significantly on station distribution. Observations at several key azimuths with respect to source strike are most sensitive to this tradeoff, and we show preliminary results that suggest weighting data based on even azimuthal distribution can improve inversion results. The tradeoff also depends on uncertainties in modeled phase amplitudes; for the USGS W phase solution of the Tohoku earthquake, this can account for errors of $\approx \pm 0.04$ in magnitude, and $\approx \pm 2^{\circ}$ in dip. Our results suggest that these uncertainties can also be reduced by weighting data according to the azimuths of optimal expected amplitudes, though more work is needed to fully quantify the improvements offered by such a scheme, and the optimal weights required. The moment-dip tradeoff also shows significant sensitivity to source depth, for which $\pm 5 \mathrm{~km}$ uncertainties can account for errors of $\approx \pm 13 \%$ in both moment and dip in the USGS W phase solution for the Tohoku earthquake. Such errors can be reduced, however, by incorporating a priori information from models such as the USGS Slab1.0 subduction interface compilation, for subduction zone earthquakes.

[20] By constraining the $\mathrm{W}$ phase inversion for the Tohoku earthquake to the Pacific slab interface model, we derive results that are consistent with other CMT inversions for this event, consistent with analyses of the earthquake using finite fault inversion techniques and, perhaps more importantly, that inherently match the regional tectonic framework. Ultimately, our preferred solution (Figure 3 ) has a moment of $4.2 \times 10^{29}$ dyne-cm $\left(\mathrm{M}_{\mathrm{w}} 9.02\right)$, and a dip of $12^{\circ}$. We also performed a double-couple inversion while fixing the strike to be $195^{\circ}$ according to the USGS Slab1.0 model. The resulting solution is similar to the preferred, with the same 
scalar moment and strike $/$ dip $/$ rake $=195^{\circ} / 12^{\circ} / 84^{\circ}$. We suggest that a reasonable range of solutions resulting from the moment-dip tradeoff lead to magnitude estimates of $\mathrm{M}_{\mathrm{w}}$ 9.01-9.11, and dips of $8^{\circ}-13^{\circ}$. As proposed by Hayes et al. [2011], these estimates verify that the difference between the USGS W phase and gCMT solutions for this earthquake can be explained by the moment-dip tradeoff. Implementation of the approaches outlined in this paper can help reduce the moment-dip tradeoff in CMT inversions for future earthquakes, and help better-quantify the true moments and dips of these events.

[21] Acknowledgments. We thank L. Rivera for providing scripts and other support, and two anonymous reviewers for comments that lead to improvements in the paper. This work was partially supported by a Mendenhall Postdoctoral Fellowship to VCT.

[22] The Editor wishes to thank two anonymous reviewers for their assistance evaluating this paper.

\section{References}

Abercrombie, R. E., M. Antolik, K. Felzer, and G. Ekström (2001), The 1994 Java tsunami earthquake: slip over a subducting seamount, J. Geophys. Res., 106, 6595-6607, doi:10.1029/2000JB900403.

Alterman, Z., H. Jarosch, and C. L. Pekeris (1959), Fast evaluation of source parameters from isolated surface-wave signals, Bull. Seismol. Soc. Am., 60, 1337-1387.

Ben-Menahem, A., M. Rosenman, and D. G. Harkrider (1970), Oscillations of the earth, Proc. R. Soc. London, Ser. A, 252, 80-95.

Dahlen, F. A. (1993), Single-force representation of shallow landslide sources, Bull. Seismol. Soc. Am., 83, 130-143.

Duputel, Z., L. Rivera, H. Kanamori, G. P. Hayes, B. Hirshorn, and S. Weinstein (2011), Real-time W phase inversion during the 2011 off the Pacific coast of Tohoku earthquake, Earth Planets Space, 63, 535-539.

Dziewonski, A. M., and D. L. Anderson (1981), Preliminary reference Earth model, Phys. Earth Planet. Inter., 25, 297-356, doi:10.1016/ 0031-9201(81)90046-7.

Dziewonski, A. M., T. A. Chou, and J. H. Woodhouse (1981), Determination of earthquake source parameters from waveform data for studies of global and regional seismicity, J. Geophys. Res., 86, 2825-2852, doi:10.1029/JB086iB04p02825.

Ekström, G., A. M. Dziewonski, N. N. Maternovskaya, and M. Nettles (2005), Global seismicity of 2003: Centroid-moment-tensor solutions for 1087 earthquakes, Phys. Earth Planet. Inter., 148, 327-351, doi:10.1016/j.pepi.2004.09.006.

Engdahl, E. R., R. van der Hilst, and R. Buland (1998), Global teleseismic earthquake relocation with improved travel times and procedures for depth determination, Bull. Seismol. Soc. Am., 88, 722-743.

Fox, J. (1997), Applied Regression Analysis, Linear Models, and Related Methods, 624 pp., Sage, Thousand Oaks, Calif.

Hashimoto, C., K. Fukui, and M. Matsu'ura (2004), 3-D modeling of plate interfaces and numerical simulation of long-term crustal deformation in and around Japan, Pure Appl. Geophys., 161, 2053-2068, doi:10.1007/ s00024-004-2548-8.
Hayes, G. P. (2011), Rapid source characterization of the 2011 Mw 9.0 off the Pacific coast of Tohoku earthquake, Earth Planets Space, 63, 529-534.

Hayes, G. P., D. J. Wald, and K. Keranen (2009), Advancing techniques to constrain the geometry of the seismic rupture plane on subduction interfaces a priori: Higher order functional fits, Geochem. Geophys. Geosyst., 10, Q09006, doi:10.1029/2009GC002633.

Hayes, G. P., P. S. Earle, H. M. Benz, D. J. Wald, and R. W. Briggs, and the USGS/NEIC Response Team (2011), 88 hours: The U.S. Geological Survey National Earthquake Information Center response to the 11 March 2011 Mw 9.0 Tohoku earthquake, Seismol. Res. Lett., 82, 481-493, doi:10.1785/gssrl.82.4.481.

Hjorleifsdottir, V., and G. Ekström (2010), Effects of three-dimensional Earth structure on CMT earthquake parameters, Phys. Earth Planet. Inter., 179, 178-190, doi:10.1016/j.pepi.2009.11.003.

Kagan, Y. Y. (2003), Accuracy of modern global earthquake catalogs, Phys. Earth Planet. Inter., 135, 173-209, doi:10.1016/S0031-9201(02) 00214-5.

Kanamori, H. (1993), W phase, Geophys. Res. Lett., 20, 1691-1694, doi:10.1029/93GL01883.

Kanamori, H. (2006), Seismological aspects of the December 2004 Great Sumatra-Andaman earthquake, Earthquake Spectra, 22, S1-S12, doi:10.1193/1.2201969.

Kanamori, H., and J. J. Cipar (1974), Focal process of the great Chilean earthquake May 22, 1960, Phys. Earth Planet. Inter., 9, 128-136, doi:10.1016/0031-9201(74)90029-6.

Kanamori, H., and J. W. Given (1981), Use of long-period surface waves for rapid determination of earthquake-source parameters, Phys. Earth Planet. Inter., 27, 8-31, doi:10.1016/0031-9201(81)90083-2.

Kanamori, H., and L. Rivera (2008), Source inversion of W phase: Speeding up seismic tsunami warning, Geophys. J. Int., 175, 222-238, doi:10.1111/j.1365-246X.2008.03887.x.

Kanamori, H., and G. S. Stewart (1976), Mode of the strain release along the Gibbs fracture zone, Mid-Atlantic Ridge, Phys. Earth Planet. Inter., 11, 312-332, doi:10.1016/0031-9201(76)90018-2.

Konca, A. O., et al. (2007), Rupture kinematics of the $2005 \mathrm{Mw} 8.6$ NiasSimeulue earthquake from the joint inversion of seismic and geodetic data, Bull. Seismol. Soc. Am., 97, S307-S322, doi:10.1785/0120050632.

Miura, S., N. Takahashi, A. Nakanishi, T. Tsuru, S. Kodaira, and Y. Kaneda (2005), Structural characteristics off Miyagi forearc region, the Japan Trench seismogenic zone, deduced from a wide-angle reflection and refraction study, Tectonophysics, 407, 165-188, doi:10.1016/j. tecto.2005.08.001.

Park, J., et al. (2005), Earth's free oscillations excited by the 26 December 2004 Sumatra-Andaman earthquake, Science, 308, 1139-1144, doi:10.1126/science. 1112305.

Tsai, V. C., M. Nettles, G. Ekström, and A. M. Dziewonski (2005), Multiple CMT source analysis of the 2004 Sumatra earthquake, Geophys. Res. Lett., 32, L17304, doi:10.1029/2005GL023813.

Z. Duputel, Institut de Physique du Globe de Strasbourg, 5, rue René Descartes, F-67084 Strasbourg CEDEX, France.

G. P. Hayes, Geologic Hazards Science Center, U.S. Geological Survey, PO Box 25046, MS 966, Golden, CO 80225, USA.

V. C. Tsai, Seismological Laboratory, California Institute of Technology, 1200 E. California Blvd., MS 252-21, Pasadena, CA 91125, USA. (tsai@ caltech.edu) 